\title{
Innovative delivery method using a detachable device to deliver a large polyglycolic acid sheet to a gastric ulcer perforation
}
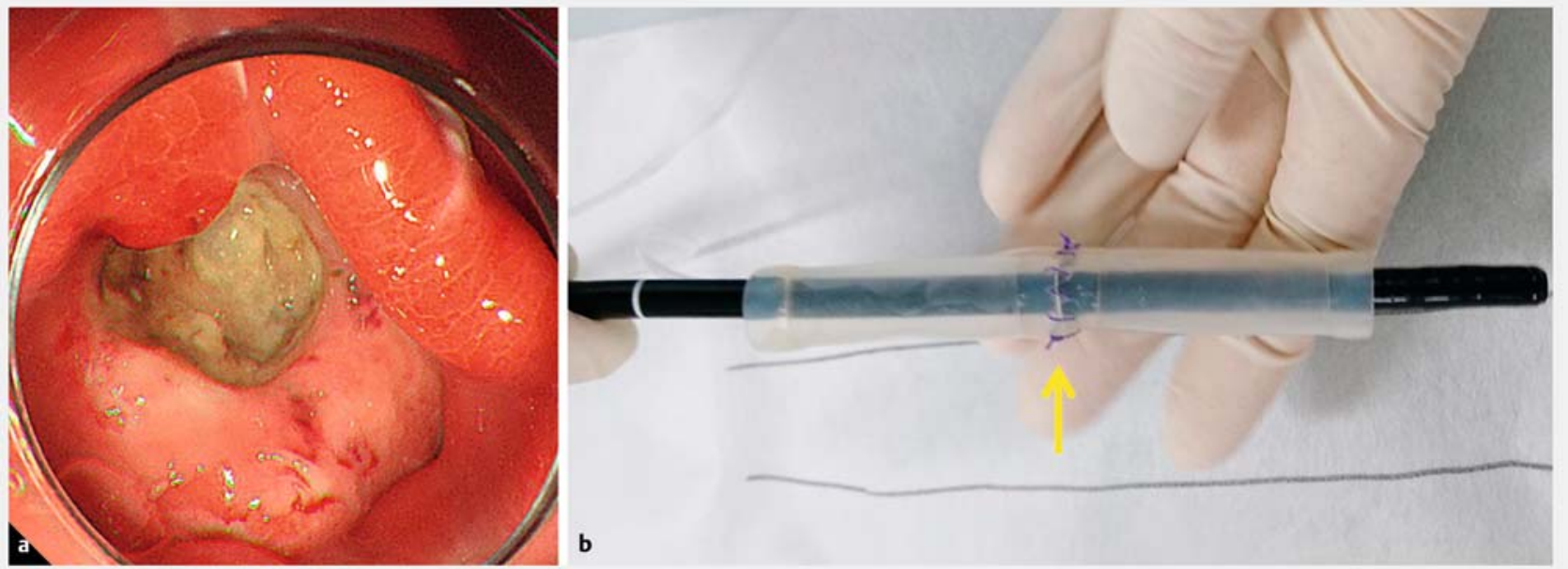

- Fig. 1 Perforation site before and after treatment with polyglycolic acid sheet. a The perforation was caused by cytomegalovirus (CMV) infection after endoscopic submucosal dissection for early gastric cancer. CMV pp 65 antigen (C7-HRP) was positive. b At 7 days after treatment, the perforation site was completely covered with regenerative tissue (yellow circle), and edematous mucosa around the ulcer was improved.

Many reports have indicated the efficacy of polyglycolic acid (PGA) sheets with fibrin glue for the treatment of perforations or preventing stricture following endoscopic submucosal dissection (ESD) $[1,2]$. However, the delivery method of such a thin membrane such as the PGA sheet (especially large sheets) into the stomach without getting wet has not been reported.

An 89-year-old woman was diagnosed with early gastric cancer. Following endoscopic submucosal dissection, a perforation was induced by cytomegalovirus infection ( $\triangleright$ Fig. $\mathbf{1}$ a), and accompanied by severe immune suppression due to oral administration of methotrexate for rheumatoid arthritis. A drip infusion of ganciclovir was administered for the infection. For treatment of the perforation, we developed a novel detachable device to deliver a large, $16 \mathrm{~cm}^{2}$ PGA sheet to completely cover the perforation ( $\vee$ Video 1$)$. Two balloons (endoscopic injection sclerotherapy balloons, $11 \mathrm{~mm}$ in diameter, $50 \mathrm{~mm}$ in length; TOP Co., Tokyo, Japan) were connected by sewing togeth-

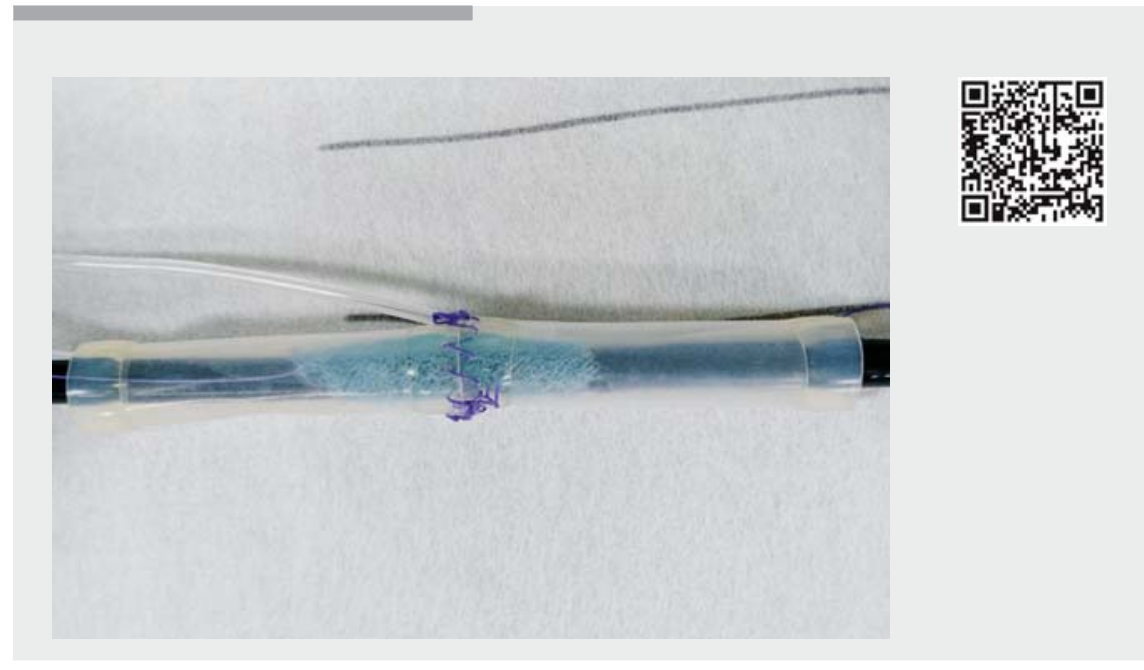

$\checkmark$ Video 1 Innovative concept of detachable device delivery system for delivery of a large polyglycolic acid (PGA) sheet to a perforation in an endoscopic submucosal dissection (ESD) ulcer. The post-ESD ulcer floor perforation was induced by cytomegalovirus infection. Two balloons (endoscopic injection sclerotherapy balloons) were sewn together to connect them and to create a sealed space. A nasal endoscope $(5 \mathrm{~mm})$ was inserted through the balloons $(11 \mathrm{~mm})$, leaving a $3 \mathrm{~mm}$ concentric circular gap, into which the PGA sheet was placed. Insufflation of air into the balloons resulted in the PGA sheet being completely sealed at the junction of the two balloons. In the stomach, the balloons were deflated and the endoscope was retracted, leaving the balloons with the PGA sheet at the perforation site. The PGA sheet was then placed over the perforation site. This novel delivery system allowed the large PGA sheet to be delivered to the perforation site without getting wet. 

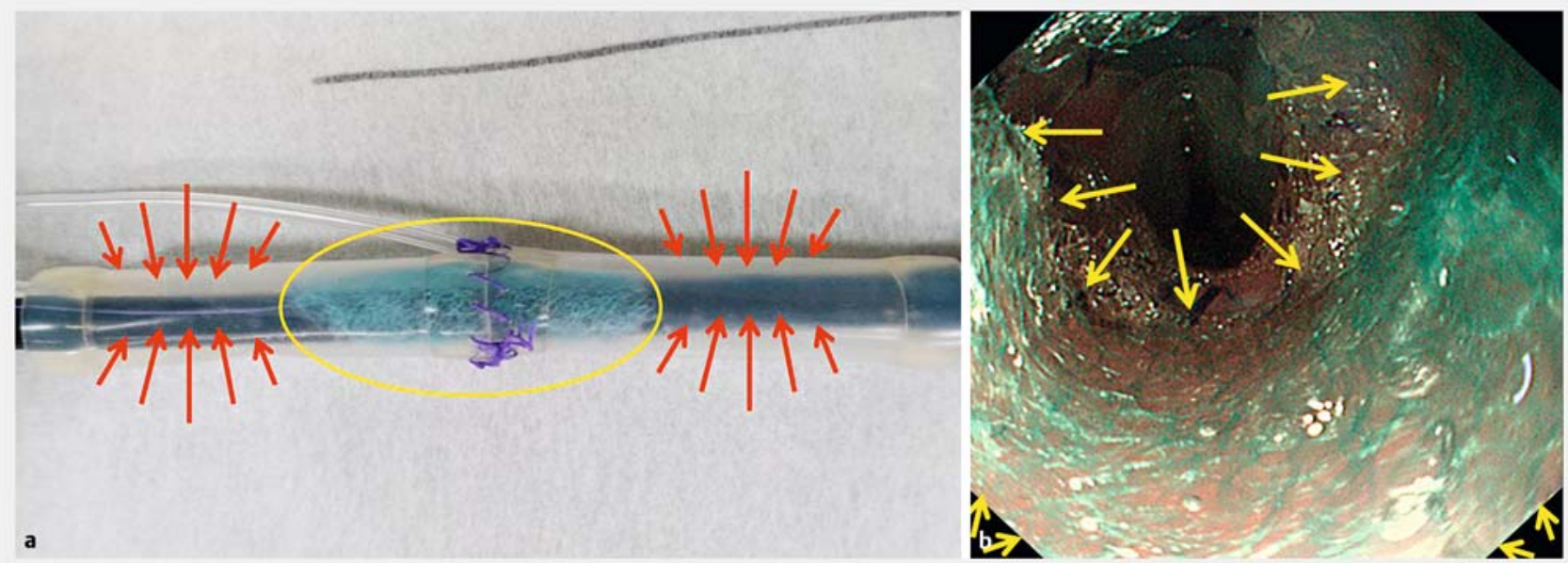

Fig. 2 Creation of polyglycolic acid (PGA) sheet delivery system. a Two balloons (endoscopic injection sclerotherapy balloons, $11 \mathrm{~mm}$ in diameter, $50 \mathrm{~mm}$ in length; TOP Co., Tokyo, Japan) were sewn together to create a sealed space underneath the junction of two balloons (yellow arrows). b A nasal endoscope ( $5 \mathrm{~mm}$ in diameter) was inserted into the balloons, leaving a $3 \mathrm{~mm}$ concentric circular gap, into which the PGA sheet was placed. Insufflation of air into the balloons (red arrows) resulted in the PGA sheet being completely sealed at the junction of the two balloons (yellow circle).
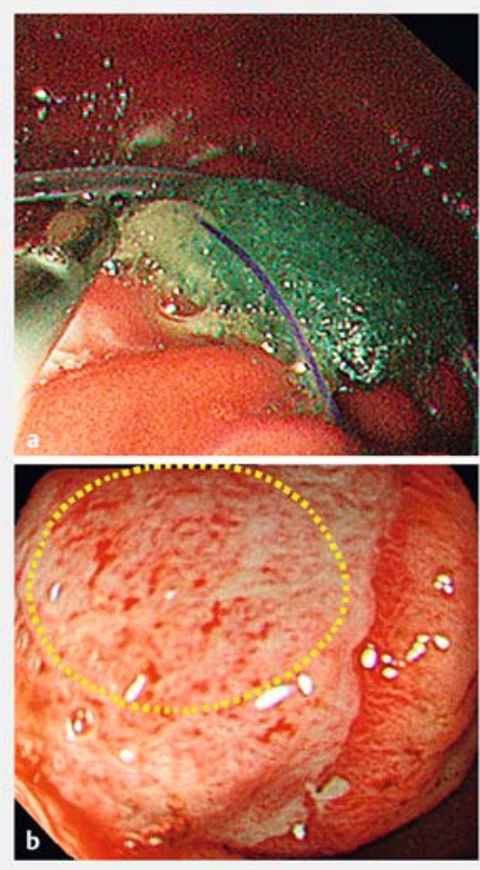

Fig. 3 Placement of the polyglycolic acid (PGA) sheet at the perforation site. a The nasal endoscope with the PGA sheet was inserted into the stomach to the site of the perforation. The balloons were deflated and the endoscope was retracted, leaving the balloons with the PGA sheet at the perforation site. The large $16 \mathrm{~cm}^{2}$ PGA sheet within the deflated balloons can be seen (yellow arrows). $\mathbf{b}$ The grasping thread attached to the PGA sheet was then used to pull the sheet from underneath the balloons, to enable final placement over the perforated artificial ulcer. er ( $\triangleright$ Fig. 2 a). A nasal endoscope ( $5 \mathrm{~mm}$ in diameter) was inserted through the balloons, leaving a $3 \mathrm{~mm}$ diameter concentric circular gap, into which the PGA sheet was placed. Insufflation of air into the balloons resulted in the PGA sheet being completely sealed at the junction of the two balloons ( $\mathbf{F i g} \mathbf{2} \mathbf{b}$ ). The nasal endoscope with the PGA sheet was then inserted into the stomach to the site of the perforation. The balloons were deflated and the endoscope was retracted, leaving the balloons with the PGA sheet at the perforation site ( $\mathbf{F i g . 3} \mathbf{a}$ ). The grasping thread attached to the PGA sheet was then used to pull the sheet from underneath the balloons ( $>$ Fig. $\mathbf{3 b}$ ), to enable final placement of the PGA sheet over

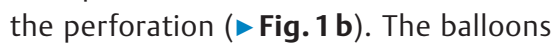
were subsequently removed by re-inserting the nasal endoscope through the balloons, inflating the balloons, and then retracting the endoscope, with the balloons attached.

Although Tsuji et al. reported a new delivery method for PGA sheets using a normal-caliber endoscope and a small-caliber endoscope inside an overtube [3], this method is rather difficult to maneuver. The current innovative concept of detachable delivery system is useful for the delivery of large PGA sheets in the digestive tract, including the lower tract, especially in proximal colon or cecal ESD.
Endoscopy_UCTN_Code_TTT_1AO_2AI

Competing interests

None

The Authors

Hirohito Mori ${ }^{1}$, Hideki Kobara ${ }^{1}$, Asadur Rahman², Noriko Nishiyama', Akira

Nishiyama², Yasuyuki Suzuki ${ }^{3}$, Tsutomu Masaki $^{1}$

1 Department of Gastroenterology and Neurology, Kagawa University, Kagawa, Japan

2 Department of Pharmacology, Kagawa University, Kagawa, Japan

3 Department of Gastroenterological Surgery, Kagawa University, Kagawa, Japan

\section{Corresponding author}

\section{Hirohito Mori, MD, PhD}

Department of Gastroenterology and Neurology, Kagawa University, 1750-1 Ikenobe, Miki, Kita, Kagawa 761-0793, Japan Fax: +81-87-8912158

hiro4884@med.kagawa-u.ac.jp 


\section{References}

[1] Takimoto K, Toyonaga T, Matsuyama K. Endoscopic tissue shielding to prevent delayed perforation associated with endoscopic submucosal dissection for duodenal neoplasms. Endoscopy 2012; 44: E1 -3

[2] Sakaguchi Y, Tsuji Y, Ono S et al. Polyglycolic acid sheets with fibrin glue can prevent esophageal stricture after endoscopic submucosal dissection. Endoscopy 2015; 47 : $336-340$
[3] Tsuji K, Doyama H, Nakanishi H et al. A new technique for delivering a polyglycolic acid sheet to cover a large mucosal defect: the Swiss roll method. Endoscopy 2014; 46: E547-548

\section{Bibliography}

DOI https://doi.org/10.1055/s-0043-105573

Endoscopy 2017; 49: E165-E167

(C) Georg Thieme Verlag KG

Stuttgart · New York

ISSN 0013-726X
ENDOSCOPY E-VIDEOS

https://eref.thieme.de/e-videos

口7f: Endoscopy E-Videos is a free

access online section, reporting

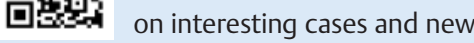

techniques in gastroenterological endoscopy. All papers include a high quality video and all contributions are freely accessible online.

This section has its own submission website at

https://mc.manuscriptcentral.com/e-videos 